\title{
An Explanation of the Anomalous Magnetic Moment of Electron and of Muon in Accordance with a Pre-Quantum Vortexial Model of Particle and of Magnetic Moment
}

\author{
Marius Arghirescu \\ State Office for Inventions and Trademarks, OSIM, Romania \\ Email: arghirescu.marius@osim.ro
}

\begin{abstract}
In the paper is presented a possible classical explanation of the anomalous value $a_{e}=\alpha / 2 \pi$ of the electron' and of the muon's magnetic moment, based on a vortexial pre-quantum model of electron and on the vortexial nature of the electron's magnetic moment resulted in the model as etherono-quantonic vortex: $\Gamma_{\mu}^{*}(\mathrm{r})=\Gamma_{\mu}\left(\mathrm{r}_{\mu}{ }^{\prime}\right)+\Gamma_{\mathrm{B}}\left(\mathrm{r}>\mathrm{r}_{\mu}{ }^{\prime}\right)$, of ,heavy' etherons $\left(\mathrm{m}_{\mathrm{s}} \approx 10^{-60} \mathrm{~kg}\right)$ - generating the magnetic potential $\mathbf{A}$ and of quantons $\left(\mathrm{m}_{\mathrm{h}}=\mathrm{h} \cdot 1 / \mathrm{c}^{2}=7.37 \times 10^{-51} \mathrm{~kg}\right)$ - generating vortex-tubes $\boldsymbol{\xi}_{\mathrm{B}}$ that materializes the $\mathbf{B}$-field lines of the magnetic induction and a spinorial mass $\mathrm{m}_{\mu}$ of photons vortexed with the light' speed in the volume of radius $r_{\mu}{ }^{\prime}=r_{\mu}{ }^{0}(1+\alpha / 2 \pi),\left(r_{\mu}^{0}=h / m_{e} c\right)$. The value of the spinorial $m_{\mu}$-mass which explains the anomalous magnetic moment results almost equal but lower than the inertial mass $m_{e}$ to which it not contribute, by the conclusion that the quantonic $\Gamma^{*}{ }_{\mu}(\mathrm{r})$ vortex generates the inertial $\mathrm{m}_{\mathrm{e}^{-}}$mass by photons confining with only a part $\Delta \Gamma_{\mathrm{B}}$ of the $\Gamma_{\mathrm{B}}$-vortex,

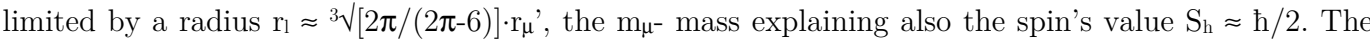
muon's and the proton's anomalous magnetic moments are expained by a composite fermion type of particle, with the e-charge given by an electron attached to a neutral cluster of magnetically paired quasi-electrons, with degenerate magnetic moment resulted by a degenerate Compton radius, i.e., decreased proportional with the density of particle's quantum volume in which is introduced the electron's superdense kernel.
\end{abstract}

Keywords: anomalous magnetic moment, Compton radius, classic electron model, particle' self-energy, muon's magnetic moment

\section{Introduction}

In the quantum electrodynamics, the anomalous magnetic moment of a particle is a contribution of effects of quantum mechanics to the magnetic moment of the particle, expressed by Feynman diagrams with loops and is usually expressed in terms of the g-factor, in the form: $a_{e}=(g-2) / 2$, the value $g=2$ corresponding to the Dirac's equation prediction. By the calculation of the vertex function for the quantum mechanical correction to the electron's magnetic moment, it was found (J. Schwinger, 1948 [1]) that:

where $\alpha$ is the fine structure constant.

$$
a_{e}=\frac{\alpha}{2 \pi} ; \alpha=1 / 137,
$$

The corrected experimentally obtained value is [2]: $a_{e}=0.00115965218073(28)$.

The magnetic moment $e \hbar / 2 m$ of the Dirac electron results by a circular motion of radius equal to the reduced Compton wavelength (the Compton radius): $\lambda=r_{\mu}=\hbar / m_{e} c,[3],(\hbar=h / 2 \pi)$.

It is considered also that the mass of the electron has a spatial distribution extended towards infinite and exists experimental evidence for the electromagnetic origin of the mass of a charged particle [4].

A classical deduction of the anomalous magnetic moment of the electron was recently made by considering the rotation of the electron's charge with the light' speed around its mass center, with charge' and mass' corrections resulted by the zero-point energy and by conclusion that the mass of the particle may be interpreted to the zero-point field energy associated with the local complex rotation or oscillation, confined in a region of space of the order of the Compton wavelength, [5]. 
Another theoretic deduction of the value $a_{e}=\alpha / 2 \pi$ was made by A. O. Barut and colab. [6] which considered the anomalous value $\mathrm{a}_{\mathrm{e}}$ as effect of the particle's self-fields and not of electromagnetic vacuum fluctuations.

The anomalous magnetic moment of the muon is calculated in a similar way, the prediction of the quantum electrodynamics being [7]: $a_{\mu}=0.00116591804(51)$. The experimentally obtained value is [7] $a_{\mu}=$ $0.0011659209(6)$.

In a Cold Genesis Theory of Matter and Fields of the author, (CGT-[8-10]), based on the Galilean relativity, the discovered elementary particles are explained by a vortexial model, of composite fermion type, as non-destructive collapsed Bose-Einstein Condensate of $1 \frac{1}{2} N^{p}$ gammons considered as thermalized pairs: $\tilde{\gamma}^{*}=\left(e^{-} e^{+}\right)$of axially coupled electrons with opposed charges which became degenerate electrons inside the $\mathrm{N}^{\mathrm{p}}$ cluster, i.e- quasi-electrons with diminished mass, charge and magnetic moment: $m_{e}{ }^{*} \approx 0.81$ $m_{e} ; e^{*} \approx(2 / 3) e ; \mu_{e}^{*} \approx \mu_{e}\left(2.79 m_{e} / m_{P}\right) \approx \mu_{P},[9,10]$.

The CGT uses an electron model with the charge $e=S^{0} / k_{1}$ contained by its surface $S^{0}=4 \pi a^{2}$ of radius: $a=1.41 \mathrm{fm}$, (close to the value of the nucleon radius resulted from the expression of the nuclear volume: $r_{n} \approx 1.25 \div 1.45 \mathrm{fm}$ ) and with an exponential variation of its density and of quanta density variation inside the electron's quantum volume:

$$
\begin{gathered}
\rho_{e}=\rho_{e}^{0} \cdot e^{-r / \eta} ;\left(\rho_{e}^{0}=22.24 \mathrm{~kg} / \mathrm{m}^{3} ; \eta=0.965 \mathrm{fm}\right) ; \\
\rho_{e}(a)=\mu_{0} / k_{1}{ }^{2}=5.17 x 10^{-13} \mathrm{~kg} ;\left(k_{1}=4 \pi \cdot a^{2} / e\right)
\end{gathered}
$$

It may be argued that the E-field quanta (vectorial photons, "vectons"-in CGT) results from pseudoscalar quanta of background radiation of $\sim 2.73 \mathrm{~K}$, the vectonic centroid's chirality giving the echarge's sign, and have a spherical distribution for $r \geq a$, which is generated by the vortexial energy of the electron's magnetic moment as consequence of the precession movement and a cylindrical density variation inside the electron, transformed into spherical variation:

$$
\begin{gathered}
\rho_{v}(r)=\rho_{v}^{0}\left(r_{v}^{0} / r\right) \text { for } r_{v}^{0}<r \leq a ; \rho_{v}(r)=\rho_{e}(a) \cdot(a / r)^{2} \text { for } r>a, \\
\rho_{v}^{0}=\rho_{e}(a) \cdot\left(a / r_{v}^{0}\right)=\rho_{e}^{0} \cdot e^{-r / \eta} \Rightarrow r_{v}^{0}=0.64 \mathrm{fm}
\end{gathered}
$$

The particle's magnetic moment $\mu_{e}^{*}$ results in CGT by an etherono-quantonic vortex: $\Gamma^{*} \mu(r)=\Gamma_{\mathrm{A}}+\Gamma_{\mathrm{B}}$ of heavy ("sinergonic") etherons $\left(m_{s} \approx 10^{-60} \mathrm{~kg}\right.$ )- generating the magnetic potential A and of quantons $\left(m_{h}\right.$ $=h \cdot 1 / c^{2}=7.37 \times 10^{-51} \mathrm{~kg}$ ) - generating vortex-tubes $\boldsymbol{\xi}_{\mathrm{B}}$ that materializes the $\mathbf{B}$-field lines of the magnetic induction, the total vortex $\Gamma_{\mu}^{*}(r)$ having a part $\Gamma_{\mu}\left(r_{\mu}\right)=2 \pi c \cdot r_{\mu},\left(r_{\mu}=\hbar / m_{p} c\right)$ which gives the particle's magnetic moment and an evanescent part $\Gamma_{\mathrm{B}}(r),\left(r>r_{\mu}\right)$ which generates the B-field.

The virtual radius: $r_{\mu}{ }^{n}$ of the proton's magnetic moment, $\mu_{p}$, results by a degenerate Compton radius of an attached positron, which is defined microphysically as the radius until which the quantons of the $\Gamma_{\mu}(r)$ vortex have still the light' speed c and which decreases when the protonic positron is included in the neutral $N^{p}$ cluster volume, from the value: $r_{\mu}{ }^{e}=3.86 \times 10^{-13} \mathrm{~m}$, to the value: $r_{i}=r_{\mu}{ }^{p}=0.59 \mathrm{fm}$, as consequence of the increasing of the impenetrable quantum volume mean density in which is included the protonic positron centroid: $m_{0}$, from the value: $\bar{\rho}_{e}$ to the value: $\bar{\rho}_{n} \cong f_{d} \cdot N^{p} \cdot \bar{\rho}_{e}$, in which: $k_{P}=\bar{\rho}_{n} / \bar{\rho}_{e}$ -the gyromagnetic ratio; $\bar{\rho}_{e} ; \bar{\rho}_{n}$-the mean density of electron and of nucleon; $f_{d} \approx 0.81$-the degeneration coefficient which gives the quasielectron's mass, $m_{e}{ }^{*} \approx 0.81 m_{e}$.

The superposition of the $\left(N^{p}+1\right)$ quantonic vortices: $\Gamma_{\mu}{ }^{*}$ of the protonic quasielectrons generates inside a volume with the radius: $r_{\mu}{ }^{a}=2.35 \mathrm{fm}$ a total dynamic pressure: $P_{n}=\left({ }^{1 /}{ }_{2}\right) \rho_{n}(r) \cdot c^{2}$ which gives a nuclear potential: $\mathrm{V}_{n}(r)$, in an eulerian form, having a variation according to eqn.:

$$
V_{n}(r)=-v_{i} P_{n}(r)=-V_{n}^{0} \cdot e^{-r / \eta^{*}} ; V_{n}^{0}=v_{i} P_{n}^{0}=\left({ }^{v i} / 2\right) \rho_{n}{ }^{0} \cdot c^{2} \text { with: } \eta^{*}=0.8 \mathrm{fm},
$$

$\left(v_{i}(0.6 \mathrm{fm}) \approx 0.9 \mathrm{fm}^{3}\right.$ - the impenetrable quantum volume of the nucleon, $\mathrm{V}_{n}{ }^{0}=109.8 \mathrm{MeV}$-the potential well) which explains the strong force in correlation with the brownian energy of some internal ,naked' photons which explains the repulsive shell of the impenetrable nucleonic volume.

Also, the neutron results in CGT by a specific "dynamide" model, with a degenerate electron with degenerate magnetic moment: $\mu_{e}^{s} \approx-4.6 \mu_{N}\left(\mu_{N}\right.$ - the nuclear magneton), rotated inside the quantum volume of a proton by the etherono-quantonic vortex $\Gamma_{P}$ of the protonic magnetic moment $\mu_{p}$.

An important experimental argument in the favor of the composite fermion particle model of CGT is the almost same size order of the radius of scattering centers determined inside the electron and inside 
the nucleon, $\left(\sim 10^{-18} \mathrm{~m}[11]\right.$ - value considered also for quarks [12], but being the radius of a super-dense electronic kernel (centroid), in CGT, [8-10]);

A question that arises is if a classical pre-quantum model of electron may explain the anomalous magnetic moment of the electron and of the muon.

\section{The Anomalous Magnetic Moment's Explaining by a Pre-Quantum Model of Electron}

Classically, according to a cold barrel-like electron model of electron, the magnetic moment of the electron may be found considering a rotation of the charge e contained by a cylindrical surface of radius $r_{\mu}=r_{\lambda}$ and high $h \geq 2 a_{e}\left(a_{e}\right.$-the classical radius of the electron with superficial distribution of the charge $e$ ) with the light speed $c$, according to the relations:

$$
\mu_{e}=i x S_{\mu}=\frac{e}{\tau} S_{\mu}=\frac{e \cdot c}{2 \pi \cdot r_{\mu}} \pi \cdot r_{\mu}^{2}=\frac{e \cdot c \cdot r_{\mu}}{2}=\frac{e}{4 \pi \cdot m_{e} c}=\frac{e}{m_{e}} S_{h} ; \mathrm{r}_{\mu}=\mathrm{r}_{\lambda}=\frac{\lambda_{\mathrm{e}}}{2 \pi}=\frac{\hbar}{m_{e} c} ; \mathrm{S}_{h}=\frac{\hbar}{2}
$$

According to eqn. (3), the anomalous magnetic moment of the electron may result by a little increased value of the magnetic moment's radius: $r_{\mu}^{\prime}>r_{\mu}^{0}=\hbar / m_{e} c,(\hbar=h / 2 \pi$, $h$-the Planck's constant $)$.

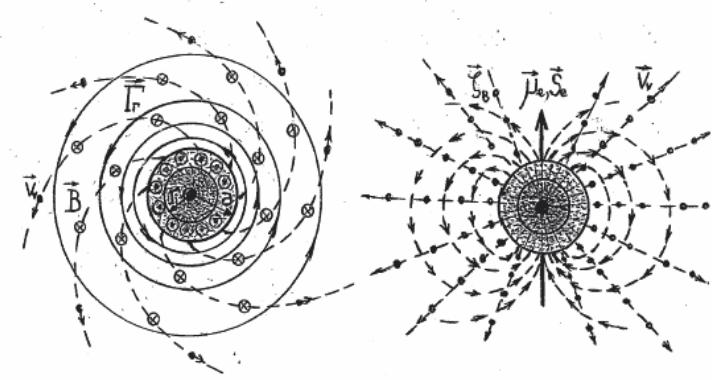

Figure 1. Chiral pre-quantum model of electron (CGT)

The appearance of the $e$ - charge's rotation with the light speed c to a vortex circle of radius $r_{\mu}\left(l_{\mu}=\right.$ $2 \pi r_{\mu}$ ) results in CGT by the fact that the value of the $e$-charge is given by the flux of the E-field's quanta:

$$
\phi_{e}=4 \pi r^{2} c \rho_{e}(a) \cdot(a / r)^{2}=4 \pi a^{2} c \rho_{e}(a) ; e=\varepsilon_{0} 4 \pi a^{2} \mathrm{E}(a)=\varepsilon_{0} 4 \pi a^{2} k_{1} \rho_{e}(a) c^{2}=\varepsilon_{0} k_{1} c \cdot \phi_{e} ;\left(k_{1}=4 \pi a^{2} / e\right)
$$

and the magnetic moment $\mu_{\mathrm{e}}$ is given by the speed of the quantons of $\Gamma_{\mu}$-vortex in report with the 'vectons' of the $\phi_{e}$-flux at the limit $r=r_{\mu}$ ', these 'vectons' being considered pseudo-radially emitted, with $v_{v} \approx c$ at least from the limit $r=r_{\mu}$ and inducing the forming of vortex-tubes $\xi_{\mathrm{B}}$ that materializes the $\mathbf{B}$ field lines, according to the model [8-10], (fig. 1).

For approximate the value of $r_{\mu}^{\prime}$ we must make the next observations:

- Classically, the electron' intrinsic energy: $m_{e} c^{2}$, for the case of an e-charge contained by the electron's surface, results as equal with the total electrostatic energy, $\mathrm{E}_{\mathrm{E}}$, by the equality:

$$
m_{e} c^{2}=E_{E}=\frac{\varepsilon_{0}}{2} \int_{a}^{\infty} 4 \pi r^{2} E^{2} d r=\frac{e^{2}}{8 \pi \varepsilon_{0} a}
$$

which gives a classical radius corresponding to superficial distribution of $e$-charge: $a=a_{v} / 2=1.41 \mathrm{fm}$, half from the classical radius $a_{v}=2.82 \mathrm{fm}$, corresponding to a volumic distribution of the $e$-charge.

- From generic point of view, in a classic model, the electron's inertial mass $\mathrm{m}_{\mathrm{e}}$ results vortexially, by the confining of photons of the quantum vacuum around a superdense electronic kernel (centroid) $m_{0}$ by the vortexial energy of the electron's magnetic moment $\mu_{e}$, given by an etherono-quantonic vortex $\Gamma_{\mu}^{\prime}=$ $2 \pi c \cdot r\left(r \leq r_{\mu}{ }^{\prime}\right)$ which corresponds to a magnetic-like energy $\mathrm{E}_{\mu}$ and which may also kinetise a mass $m_{\mu}$ of light photons with an exponential spheric-symmetrical distribution of quanta density: $\rho_{v}(r)=\rho_{v}{ }^{0}(a / r)^{2}$, vortexed at the light speed $v=c$ around the inertial mass $m_{e}$ in the volume of radius $r_{\mu}^{\prime}$, and by the 
action of the magnetic energy $\mathrm{E}_{\mathrm{H}}$ generated by the electron's magnetic moment $\mu_{e}$ in the evanescent part, $\left(r>r_{\mu}{ }^{\prime}\right)$, by the etherono-quantonic vortex $\Gamma_{\mathrm{B}}{ }^{\prime}=2 \pi v_{c} \cdot r,\left(v_{c} \cdot r=c \cdot r_{\mu}{ }^{\prime}\right)$.

Also, because it is not plausible that all the energy of the $\Gamma_{\mathrm{B}}{ }^{\prime}$-vortex contributes to the inertial $m_{e}-$ mass forming, we may consider that only a closer part: $\Delta \Gamma_{\mathrm{B}}{ }^{\prime}\left(r_{l}\right)$, limited by a spherical surface of radius: $r_{l}=k_{\mathrm{H}} r_{\mu}^{\prime}\left(k_{\mathrm{H}}>1\right)$ contributes to the inertial $m_{e}$-mass forming, corresponding to a magnetic type energy:

$$
\begin{aligned}
& \Delta E_{H}=\Delta m_{H} c^{2}=\frac{1}{2 \mu_{0}} \int_{r_{\mu}^{\prime}}^{r_{l}} 4 \pi r^{2} B^{2} d r=\frac{\mu_{0} \mu_{e}^{\prime 2}}{2 \pi} \int_{r_{\mu}^{\prime}}^{r_{l}} \frac{d r}{r^{4}}=\frac{\mu_{0} \mu_{e}^{\prime 2}}{2 \pi}\left(\frac{1}{3 r_{\mu}^{\prime 3}}-\frac{1}{3 r_{\mu}^{\prime 3} k_{H}^{3}}\right)=\frac{e^{2}}{8 \pi \varepsilon_{0} r_{\mu}^{\prime}}\left(\frac{1}{3}-\frac{1}{3 k_{H}^{3}}\right) \\
& \Delta \mathrm{E}_{\mathrm{H}}=m_{e} c^{2} \cdot \frac{a}{r_{\mu}^{\prime}}\left(\frac{1}{3}-\frac{1}{3 k_{H}^{3}}\right) ; \mathrm{B}(r)=\frac{\mu_{0}}{2 \pi} \frac{\mu_{e}^{\prime}}{r^{3}} ; \mu_{\mathrm{e}}^{\prime}=\frac{e \cdot c \cdot r_{\mu}^{\prime}}{2} ; r_{1}=k_{H} \cdot r_{\mu}^{\prime}{ }_{\mu}
\end{aligned}
$$

in which $a=1.41 \mathrm{fm}, \Delta m_{\mathrm{H}}$ representing the equivalent mass of photons which may be vortexed at the speed $v_{t}=c\left(c\right.$ - the light' speed) by the etherono-quantonic vortex $\Delta \Gamma_{\mathrm{B}}{ }^{\prime}\left(r_{l}\right): \Delta m_{\mathrm{H}} c^{2}=\Delta \Sigma\left(m_{f} v_{f}^{2}\right)_{r},\left(r_{l} \geq r>\right.$ $\left.r_{\mu}^{\prime} ; v_{f} \cdot r=c \cdot r_{\mu}^{\prime}\right)$.

The expression of $\mathrm{B}$ used in eqn. (5) corresponds to those found by the classic electromagnetism and to the relation: $\mathrm{E}\left(r_{\mu}{ }^{\prime}\right)=c \cdot \mathrm{B}\left(r_{\mu}^{\prime}\right)$ specific to the value of the quanta' speed $v_{c} \equiv v_{c}{ }^{r}=c$ in the $\Gamma_{\mathrm{B}}{ }^{\prime}$-vortex in report with the E-field's quanta ('vectons') up to the limit $r=r_{\mu}$ ' and to the expression of the B-field used in CGT, for $r>r_{\mu}^{\prime}[8]$ :

$$
B(r)=k_{1} \rho_{v} \mathrm{v}_{c}^{r} \cong k_{1} \rho_{a}^{0} \frac{a^{2}}{r^{2}} \cdot \frac{r_{\mu} c}{r}=k_{1} \rho_{B} c=\frac{\mu_{0}}{2 \pi} \cdot \frac{\mu_{e}}{r^{3}} ; \rho_{a}^{0}=\frac{\mu_{0}}{k_{1}^{2}} ; \rho_{B}=\frac{\mathrm{v}_{\mathrm{v}}^{r}}{c} \rho_{v} ; r>r_{\mu}^{\prime} ;
$$

( $\rho_{\mathrm{B}}$-the density of the formed $\xi_{\mathrm{B}}$-vortex-tubes; $k_{1}=4 \pi a^{2} / e$ ).

According to the previous conclusions, the mass $m_{\mu}$ of vortexed light photons of the $\mu_{e}$-magnetic moment represents a spinorial mass which generates the electron's spin but- contrary to other classical models [17], without participation to the electron's inertial mass (as consequence of the fact that its quanta are weakly linked to the inertial mass $m_{e}$ and not impede its accelerating) and which results by the relation:

$$
m_{\mu} c^{2}=E_{\mu}=\frac{1}{2 \mu_{0}} \int_{a_{s}}^{r_{\mu}^{\prime}} 4 \pi r^{2} B_{\mu}^{2} d r=m_{e} c^{2}-\Delta m_{H} c^{2}=m_{e} c^{2}\left(1-\frac{a}{3 r_{\mu}^{\prime}}+\frac{a}{3 k_{H}^{3} r_{\mu}^{\prime}}\right)
$$

The mass $\mathrm{m}_{\mu}$ of vortexed light photons (particularly- paired 'vectons') gives the spin's value $S_{h} \approx \hbar / 2$ classically, by an impulse density of quanta: $\boldsymbol{\rho}_{v}(r) c=\boldsymbol{\rho}_{v}^{0} c(a / r)^{2}$ around the inertial mass $m_{e}$, in the volume of radius $r_{\mu}$, induced by the action of the etherono-quantonic vortex $\Gamma_{\mu}=2 \pi c \cdot r_{\mu}$ ' having a (mean) quanta' speed: $v=c$ for $r \leq r_{\mu}^{\prime}$ and $v=c \cdot\left(r_{\mu}^{\prime} / r\right)$ for $r>r_{\mu}^{\prime}$, according to the relation:

$$
S_{h}^{e}=\int_{a}^{r_{\mu}^{\prime}} d m_{\mu} \cdot r \cdot c=4 \pi \rho_{v}^{0} a^{2} c \int_{a}^{r_{\mu}^{\prime}} \frac{d r}{r^{2}} r^{3} \approx m_{\mu} c \cdot \frac{r_{\mu}^{\prime}}{2} \approx \frac{h}{4 \pi}=\frac{\hbar}{2} ; m_{\mu}=\int_{a}^{r_{\mu}^{\prime}} d m_{\mu} \approx 4 \pi \rho_{v}^{0} a^{2} r_{\mu}^{\prime}
$$

By the conclusion that the electron spin's value $\hbar / 2$ is correct, from eqns $(7)$ and (8) it results that:

$$
S_{h}^{e} \approx m_{\mu} c \cdot \frac{r_{\mu}^{\prime}}{2}=m_{e}\left[1-\frac{a}{3 r_{\mu}^{\prime}}\left(1-\frac{1}{k_{H}^{3}}\right)\right] \cdot c \frac{r_{\mu}^{\prime}}{2}=m_{e} c \cdot\left(\frac{r^{\prime}}{2}-\frac{a}{6}+\frac{a}{6 k_{H}^{3}}\right)=\frac{\hbar}{2}=m_{e} c \frac{r_{\mu}^{0}}{2} ; r_{\mu}^{0}=\frac{\hbar}{m_{e} c} ;
$$

The values $r_{\mu}{ }^{\prime}>r_{\mu}^{0}$ and $k_{\mathrm{H}}$ results from the conformity with the Quantum Mechanics by eqn. (9), i.e.:

$$
\begin{aligned}
& \frac{r_{\mu}^{\prime}}{2}=\frac{r_{\mu}^{0}}{2}+\frac{a}{6}\left(1-\frac{1}{k_{\mathrm{H}}^{3}}\right)=\frac{r_{\mu}^{0}}{2}\left[1+\frac{\alpha}{6}\left(1-\frac{1}{k_{\mathrm{H}}^{3}}\right)\right] \approx \frac{r_{\mu}^{0}}{2}\left(1+\frac{\alpha}{2 \pi}\right) ; r_{\mu}^{0}=\frac{\hbar}{m_{e} c} ; \alpha=\frac{a_{v}}{\mathrm{r}_{\mu}^{0}}=\frac{2 a}{r_{\mu}^{0}} \\
& \Rightarrow k_{\mathrm{H}} \approx \sqrt[3]{2 \pi /(2 \pi-6)}=2.82 ; r_{l}=k_{H} r_{\mu}^{\prime}=k_{H} r_{\mu}^{0}(1+\alpha / 2 \pi)
\end{aligned}
$$

A value $k_{\mathrm{H}} \rightarrow \infty$ corresponds to $a_{e} \approx \alpha / 6($ instead of $\alpha / 2 \pi)$ so the known anomalous value $a_{e} \approx \alpha / 2 \pi$ results by the fact that only a closer part: $\Delta \Gamma_{\mathrm{B}}{ }^{\prime}\left(r_{l}\right)$ (given by $r_{l}$ ) of the etherono-quantonic vortex $\Gamma_{\mathrm{B}}{ }^{\prime}(r)$ of the evanescent part $\left(r>r_{\mu}{ }^{\prime}>r_{\mu}{ }^{0}\right)$ contributes to the inertial $m_{e}$-mass forming, according to the model.

The electron's magnetic moment results in consequence as given by a Compton radius $r_{\mu}$ ' $>r_{\mu}{ }^{0}$, in the form:

$$
\mu_{e}=\frac{e \cdot c \cdot r_{\mu}^{\prime}}{2}=e \cdot c \cdot \frac{r_{\mu}^{0}}{2}\left(1+\frac{\alpha}{6}\left(1-1 / k_{H}^{3}\right)\right) \approx \mu_{e}^{0} \cdot\left(1+\frac{\alpha}{2 \pi}\right) ; \alpha=\frac{a_{\mathrm{v}}}{r_{\mu}^{0}}=\frac{2 a}{r_{\mu}^{0}}=1 / 137
$$


From eqns (5), (9) and (10) it results also that: $m_{\mu}=m_{e} \cdot[1-\alpha /(2 \pi+\alpha)]$ and $\mu_{e}$ may be deduced also in the form:

$$
\mu_{e}=\frac{e \cdot \hbar}{2 m_{\mu}}=\mu_{e}^{0} \frac{m_{e}}{m_{\mu}}=\mu_{e}^{0} \cdot\left[1-\frac{a}{3 r^{\prime}{ }_{\mu}}\left(1-\frac{1}{k_{H}^{3}}\right)\right]^{-1} \approx \mu_{e}^{0} \cdot\left[1+\frac{a}{3 r^{\prime}{ }_{\mu}}\left(1-\frac{1}{k_{H}^{3}}\right)\right]=\mu_{e}^{0} \cdot\left(1+\frac{\alpha}{2 \pi}\right)
$$

The vortexial energy $\mathrm{E}_{\mu}=m_{\mu} c^{2}$ corresponds to a magnetic-type energy of the etherono-quantonic vortex $\Gamma_{\mu}$ of the electron's magnetic moment (according to CGT), which is given by the action of etheronoquantonic winds.

Because -according to the model, this energy $\mathrm{E}_{\mu}$ generates not only the spin $S_{h}$ but also the electric charge and the electric field $\mathrm{E}_{e}$ given by vectorial photons ('vectons'- in CGT [8-10]) kinetised at $v_{f}=c$ and expelled from the quantum volume of the electron, it must be at least equal with the electrostatic energy of the volume of radius $r=r_{\mu}^{\prime}$, which by eqn. (5) has the value:

$$
E_{E}^{\mu}=m_{E} c^{2}=\frac{\varepsilon_{0}}{2} \int_{a}^{r_{\mu}^{\prime}} 4 \pi r^{2} E^{2} d r=\frac{e^{2}}{8 \pi \varepsilon_{0}}\left(\frac{1}{a}-\frac{1}{r^{\prime}{ }_{\mu}}\right)=m_{e} c^{2}\left(1-\frac{\alpha \pi}{2 \pi+\alpha}\right)=m_{\mu} c^{2}\left[1-\frac{\alpha}{2 \pi}(\pi-1)\right]
$$

It is observed that $m_{\mathrm{E}} c^{2}$ is almost equal but lower than $m_{\mu} c^{2}: m_{\mathrm{E}} \approx m_{\mu}(1-\alpha / 3)$.

The explanation of this result is in concordance with the conclusion that the magnetic-like energy $\mathrm{E}_{\mu}$ induces the electric $\mathrm{E}_{\mathrm{E}}$ energy and not inverse and with the conclusion of the model that the vortexed quanta of the electron's magnetic moment $\mu_{e}$ have the (mean) speed $v_{c}=c$ inside the volume of radius $r$ $=r_{\mu}^{\prime}$, fact which generates the relation: $\mathrm{E} \approx c \cdot \mathrm{B}_{\mu}$ for $r \leq r_{\mu}$, in accordance with the model and with the relations resulted in CGT for the electric E-field and for the magnetic or pseudo-magnetic B-field [ 8-10 ]:

$$
\begin{gathered}
E_{c}=k_{1} \rho_{f}(r) \cdot \mathrm{v}_{f}^{2}=k_{1} \rho_{a}^{0} \frac{a^{2}}{r^{2}} \cdot \mathrm{v}_{f}^{2} ; \rho_{a}^{0}=\rho_{f}(a) ; \mathrm{v}_{f}=c ; k_{1}=\frac{4 \pi \mathrm{a}^{2}}{e} ; \rho_{f}(r)=\rho_{a}^{0} \frac{a^{2}}{r^{2}} \\
B(r)=k_{1} \cdot \rho_{c}(r) \cdot v_{v} ; v_{v} \leq c
\end{gathered}
$$

in which- in the case of a static $e$-charge, $p_{f}=\rho_{f} v_{f}$ is the radial impulse density of vectorial photons of the E-field and $p_{c}=\rho_{c} \cdot v_{c}$ is the impulse density of vortexed quantons of the $\Gamma_{\mu}$-vortex which generates quantonic vortex-tubes $\xi_{\mathrm{B}}$ of the B-field by the impulse density gradient $\nabla p_{c},[8-10]$.

The relation $\mathrm{E}=c \cdot \mathrm{B} \mu$ is specific to $r \leq r_{\mu}^{\prime}$, by the equalities: $\rho_{c}=\rho_{f}$ and $v_{c}=v_{f}=c$, according to the model.

In this case, the energy density of the magnetic-like $\mathrm{B}_{\mu}$-field is (quasi)equal with the energy density of the electric E-field, according to the relation:

$$
\epsilon_{B}^{\mu}=\frac{1}{2 \mu_{0}} B^{2} \approx \frac{1}{2 c^{2} \mu_{0}} E^{2}=\frac{\varepsilon_{0}}{2} E^{2}=\epsilon_{E}^{\mu}
$$

which explains the quasi-equality between $\mathrm{E}_{\mu}=m_{\mu} c^{2}$ and $\mathrm{E}_{\mathrm{E}}{ }^{\mu}=m_{\mathrm{E}} c^{2}$ resulted according to eqn. (13).

From eqn. (8) it results also that for the composed fermions, the quantum spin $S p \approx \hbar / 2$ is only a physico-mathematical (formal) size, (conclusion concordant with those of the Quantum Mechanics), because the spinorial mass $m_{\mu}$ of a composed fermion cannot increase when its magnetic moment decreases.

Also, because CGT is based on a relativity of Galilean type, the theory deduces a relative similitude between the electron' pre-quantm model and the vectorial photon pre-quantum model, considered in a revised Munera -type of photon [10; 13], as half of a pseudo-scalar photon conceived as double vortex of sub-quanta $[10,13,14]$, with the spin $S_{f}=\hbar / 2$ resulted by a photonic spinorial mass $m_{f s}$ quasi-equal with the photon's rest mass $m_{f i}$, in concordance with the expression (8) of the classic (pre-quantum) spin, [8$10,13,14]$.

\section{The Explaining of the Anomalous Magnetic Moment of Muon and of Proton}

In the case of muon, $\left(m_{p}\left(\mu^{ \pm}\right) \approx 207 m_{e}\right.$, the anomalous value $a_{e}=\alpha / 2 \pi$ is maintained according to the relation: 


$$
\begin{aligned}
& \mu_{p}^{\prime}=\frac{e \cdot c \cdot r_{p}^{\prime}}{2}=\frac{e}{m_{\mu}} m_{\mu} c \frac{r_{p}^{\prime}}{2}=\frac{e}{m_{p}} m_{p} c \frac{r_{p}^{\prime}}{2}=\mu_{p}^{0} \cdot\left(1+\frac{\alpha}{2 \pi}\right) ; \\
& \mu_{p}^{0}=\frac{e \cdot c \cdot r_{p}^{0}}{2} ; r_{p}^{\prime} \approx r_{p}^{0} \cdot\left(1+\frac{\alpha}{2 \pi}\right) \approx \frac{m_{e}}{m_{p}} r_{\mu}^{\prime}\left(\mu_{e}^{\prime}\right) \approx r_{\mu}^{\prime} \frac{\rho_{e}}{\rho_{p}} ; r_{p}^{0}=\frac{\hbar}{m_{p} c} ;
\end{aligned}
$$

because- according to the particle model of CGT, the muon's charge is given by an attached electron (negatron or positron) with degenerate magnetic moment resulted by a degenerate Compton radius, i.e. a value decreased proportional with the density of the particle's quantum volume in which is introduced the electron' superdense kernel, (the quantum volume of the composed particles resulting the same as those of the electron in CGT [8-10]), as consequence of the fact that the vortexial energy of the $\Gamma_{\mu}^{e}-$ vortex is sheared to $N^{m} \approx 252$ degenerate electrons $\left(m_{e}{ }^{*} \approx 0.81 m_{e}\right)$ coupled with antiparallels degenerate magnetic moments $\mu_{e}^{*}$.

The anomalous magnetic moment of the proton is explained similarly to the case of the muon in CGT, but with the difference that the value 2.79 of the gyromagnetc ratio is obtained by a positioning of the protonic positron's superdense kernel at a distance $r^{+}=0.96 \mathrm{fm}$ from the proton's center, in the protonic $N^{p}$-cluster of degenerate electrons (quasielectrons), giving a Compton radius of the protonic magnetic moment, $\mu_{p}: r_{\mu}^{p}=r_{i}=0.59 \mathrm{fm}$, as consequence of an increased density of confined photons in which is included the protonic positron's centroid, conform to the equations:

$$
\mu_{p}=k_{p} \frac{m_{e}}{m_{p}} \mu_{e}=k_{p} \frac{\bar{\rho}_{e}}{\bar{\rho}_{n}} \mu_{e}=k_{p} \frac{1}{f_{d} \cdot N^{P}} \mu_{B p}=\frac{e . c \cdot r_{\mu}^{p}}{2} ; k_{p}=\frac{g_{p}}{g_{e}}=2.79=\frac{\rho_{n}\left(r^{+}\right)}{\rho_{n}^{0}}=e^{-\frac{r^{+}}{\eta d}}
$$

in which: $k_{P}$-the gyromagnetic ratio; $\bar{\rho}_{e} ; \bar{\rho}_{n}$ - the mean density of electron and nucleon;

The interpretation given by eqn. (18) of the particle mass-depending magnetic moment variation explains also the fact that- when the proton is transformed into neutron, the emitted positron regains the normal magnetic moment value $\mu_{e}$ of the free state, by the negentropy of the quantum and the subquantum medium, given by quantonic and etheronic winds, [8-10].

The virtual radius of the proton magnetic moment: $r_{\mu}{ }^{n}=0.59 \mathrm{fm}$ - resulted from eq. (18), may be considered approximately equal to the radius of the impenetrable nucleonic volume, of value: $r_{\mu}{ }^{n} \cong r_{i}\left(v_{i}\right)$ $\cong 0.6 \mathrm{fm}$ - used in the Jastrow expression of the nuclear potential, [15], by the conclusion that the impenetrable nucleonic volume being supersaturated with quantons, limitates the decreasing of the radius of protonic $\Gamma_{\mu}^{p}$ quantonic vortex, at the value: $r_{\mu}{ }^{n}=r_{i}$.

\section{Conclusions}

By a classical model of fermionic particle of CGT [8-10], based on a vortexial pre-quantum model of electron and on the vortexial nature of the electron's magnetic moment resulted in the model as etheronoquantonic vortex: $\Gamma^{*} \mu(r)=\Gamma_{\mathrm{A}}+\Gamma_{\mathrm{B}}$, of 'heavy' etherons $\left(m_{s} \approx 10^{-60} \mathrm{~kg}\right)$ and of quantons $\left(m_{h}=h \cdot 1 / c^{2}=\right.$ $7.37 \times 10^{-51} \mathrm{~kg}$ ) that generates the magnetic B-field, it results that a possible classical explanation of the anomalous value $a_{e}=\alpha / 2 \pi$ of the electron' and of the muon's magnetic moment is the generating of a spinorial mass $m_{\mu}$ of photons vortexed with the light' speed in the volume of radius $r_{\mu}^{\prime}=r_{\mu}^{0}(1+\alpha / 2 \pi)$ by the $\Gamma^{*} \mu$-vortex.

The value of the spinorial $\mathrm{m}_{\mu}$-mass which explains the anomalous magnetic moment results almost equal but lower than the inertial mass $\mathrm{m}_{\mathrm{e}}$ to which it not contribute, by the conclusion that the quantonic $\Gamma^{*} \mu(r)$ - vortex generates the inertial $m_{e^{-}}$mass by photons confining with only a part $\Delta \Gamma_{\mathrm{B}}$ of the $\Gamma_{\mathrm{B}}$-vortex, limited by a radius $r_{l} \approx \sqrt[3]{ }[2 \pi /(2 \pi-6)] \cdot r_{\mu}$, the spinorial $m_{\mu}$ mass explaining also the spin's value $S_{h} \approx \hbar / 2$.

The possibility to explain coherently the known anomalous value of the magnetic moment and the physical nature of the electron's spin only by the pre-quantum model of electron considered in CGT is an argument in the favour of this particle model and explain the fact that previous classical attempts based only to the electrostatic energy of the electron [16] couldn't give a satisfactory calculation result.

The muon's and the proton's anomalous magnetic moment is expained by a composite fermion type of particle, with the $e$-charge given by an electron attached to a neutral cluster of magnetically paired quasielectrons, with degenerate magnetic moment resulted by a degenerate Compton radius, i.e., decreased 
proportional with the density of particle's quantum volume in which is introduced the electron's superdense kernel.

\section{References}

1. Schwinger, J., "On Quantum-Electrodynamics and the Magnetic Moment of the Electron", Physical Review. 73 (4): 416-417, (1948).

2. Hanneke, D.; Fogwell, H. S.; Gabrielse, G. "Cavity Control of a Single-Electron Quantum Cyclotron: Measuring the Electron Magnetic Moment", Physical Review A. 83 (5): 052122, (2011). arXiv:1009.4831.

3. A. Messiah, Mecanique Quantique (Tome 2, chap V), Dunod, Paris (1960).

4. R. Feynman, R. Leighton, and M. Sands, Feynman Lectures in Physics, Vol.2, Mainly Electromagnetism and Matter, (Chapter 28), Addison-Wesley (1964).

5. Muralidhar, K., Prasad, M. B. R., Theory of Anomalous Magnetic Moment and Lamb Shift of Extended Electron in Stochastic Electrodynamics', Progress in Phys. Issue 4, Vol.14, (2018).

6. A. O. Barut, Jonathan P. Dowling, J. F. van Huele, 'Quantum electrodynamics based on self-fields, without second quantization: A nonrelativistic calculation of g-2', Phys. Rev. A 38, 4405, (1988)

7. Patrignani, C.; Agashe, K., "Review of Particle Physics", Chinese Physics C. IOP Publishing, 40 (10): 100001, (2016).

8. Arghirescu, M., "The material structures genesis and field effects", Ed. MatrixROM, Bucharest, (2006).

9. Arghirescu, M., "The Cold Genesis", Ed. Invel-Multimedia, (2011), viXra, 1104.0043, (2012);

Arghirescu, M., The Cold Genesis of Matter and Fields, Ed. Science PG, (2015).

10. Arghirescu, M., A Quasi-Unitary Pre-Quantum Theory of Particles and Fields and Some Theoretical Implications, IJHEP, July, 80-103, (2015).

11. R. D. Chipman, L. D. Jennings, Phys. Rev. 132 (1995) 728; CERNCOURIER, 'Precision pins down the electron's magnetism', 4 oct. (2006).

12. ZEUS Collaboration, 'Limits on the effective quark radius from inclusive e-p scattering at HERA', Physics Letters B, 757 (2016) 468-472.

13. Arghirescu, M., "A Revised Model of Photon Resulted by an Etherono-Quantonic Theory of Fields", Open Access Library Journal, 2: e1920, (2015)

14. M. Arghirescu, 'Observations Concerning the Mass Variation in a Galilean - Type Relativity', IJHEP, Vol. 5, No. 1 (2018) 44-54.

15. R. Jastrow, Phys. Rev. 81 (1951) 165.

16. N. B. Mandache, 'On the Physical Origin of the Anomalous Magnetic Moment of Electron', arXiv:1307.2063v1 [physics.gen-ph], (2013).

17. B.G. Sidharth -'Chaos Universe: From the Planck to the Hubble Scale', Nova Science Publishers, New York, (2001), 12 . 